\title{
NEOPLASIA NO SÍTIO DA COLOSTOMIA: RELATO DE TRÊS CASOS E REVISÃO DA LITERATURA
}

\author{
VALDEMIR JOSÉ ALEGRE SALLES ${ }^{1}$, PEDRO ROBERTO DE PAULA ${ }^{1}$, DEOMIR GERMANO BASSI ${ }^{1}$, MANLIO \\ BASÍLIO SPERANZINI ${ }^{I}$
}

${ }^{1}$ Departamento de Medicina da Universidade de Taubaté, São Paulo, SP, Brasil

SALLES VJA, PAULA PR, BASSI DG, SPERANZINI MB. Neoplasia no Sítio da Colostomia: Relato de Três Casos e Revisão da Literatura. Rev bras Coloproct, 2006;26(1):57-60.

RESUMO: Carcinomas raramente ocorrem no sitio da colostomia. O risco para o desenvolvimento de neoplasia maligna é semelhante a qualquer outro segmento colônico, porém se a ressecção inicial foi devida a câncer, há um significativo aumento na incidência de tumor metacrônico quando comparado à população em geral. A incidência do carcinoma metacrônico colônico é geralmente de 3 a $5 \%$. A recorrência do tumor primário, quando associada à ostomia, pode se manifestar com sangramento ou obstrução. Relatamos três doentes com tumor no sitio da colostomia, sua manifestação clínica e a conduta terapêutica adotada.

Descritores: câncer colorretal, colostomia, tumor metacrônico, complicações das colostomias.

\section{INTRODUÇÃO}

A confecção de uma colostomia, com a finalidade de desvio temporário ou definitivo do trânsito intestinal colônico, não é um procedimento isento de complicações, mesmo realizada com técnica cirúrgica adequada.

As complicações locais podem ocorrer tanto no pós-operatório imediato, precoce ou tardio, apresentando incidência variável de 15 a $30 \%$, sendo as mais frequientes: a necrose da colostomia, dermatite, abscesso, hemorragia, retração, estenose, hérnia paraostômica, prolapso, fistula colo-cutânea, perfuração para a cavidade peritoneal e mais raramente o surgimento de neoplasia ${ }^{1,2,3}$.

$\mathrm{O}$ aparecimento de neoplasia benigna ou maligna na colostomia parece estar relacionado a diversos fatores, entre os quais a metaplasia colônica secundária a uma doença inflamatória crônica ${ }^{4}$. A presença de lesão neoplásica metacrônica, a colectomia com margem de ressecção inadequada ${ }^{5}$, a polipose colônica ${ }^{2}$ e o implante ou recidiva da doença tumoral 6,7 , também são fatores que levam ao aparecimento de neoplasia na colostomia.

Diante da importância do fato e da sua baixa freqüência, relatamos 3 casos de doentes que desenvolveram lesão neoplásica na colostomia, descrevendo a sua manifestação clínica e as opções terapêuticas adotadas.

\section{RELATO DOS CASOS}

Caso 1: ECS, 27 anos, sexo feminino, negra, submetida a colectomia esquerda com colostomia

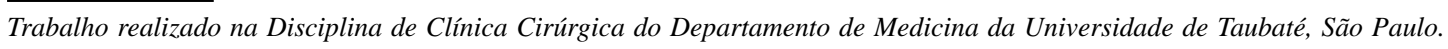

Recebido em 28/07/2005

Aceito para publicação em 26/08/2005 
terminal há dois anos, devido a neoplasia obstrutiva de cólon sigmóide. $\mathrm{O}$ exame anátomo-patológico concluiu tratar-se de adenocarcinoma tubular moderadamente diferenciado, estenosante, com invasão do tecido adiposo peri-cólico, associada à presença de extensa invasão perineural, porém não se detectando invasão vascular, com margens cirúrgicas livres e ausência de metástases em doze linfonodos mesocólicos analisados. A doente foi estadiada como Dukes B e Astler-Coller B2, sendo encaminhada ao serviço de oncologia clínica para a realização de tratamento de radioterapia e quimioterapia adjuvante.

Admitida, há dois meses, no nosso serviço com queixa de dor e sangramento na região peri-colostômica e dificuldade para a eliminação de fezes após o surgimento, de uma tumoração na colostomia que impedia a saída do efluente (Figura-1 e 2). Foi submetida a exames para o estadiamento da doença, tendo sido constatado que a doente encontrava-se com carcinomatose peritoneal.

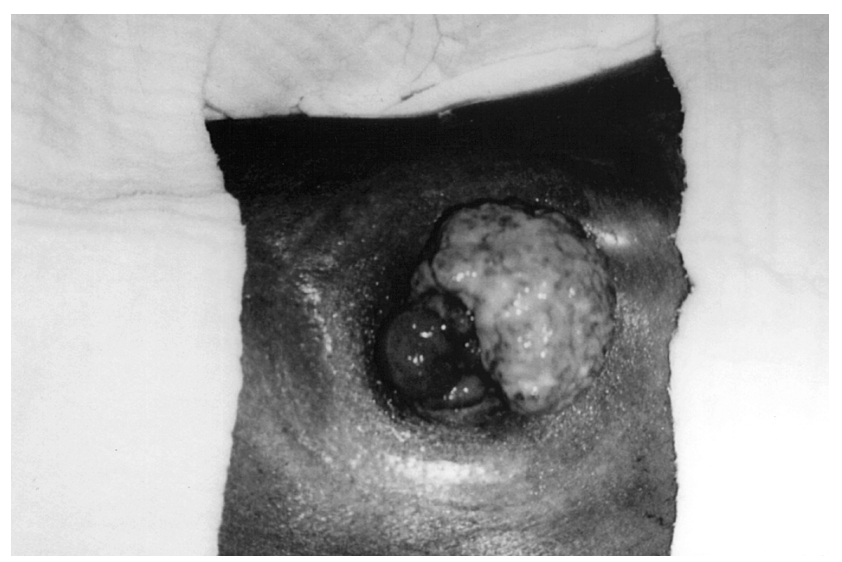

Figura 1

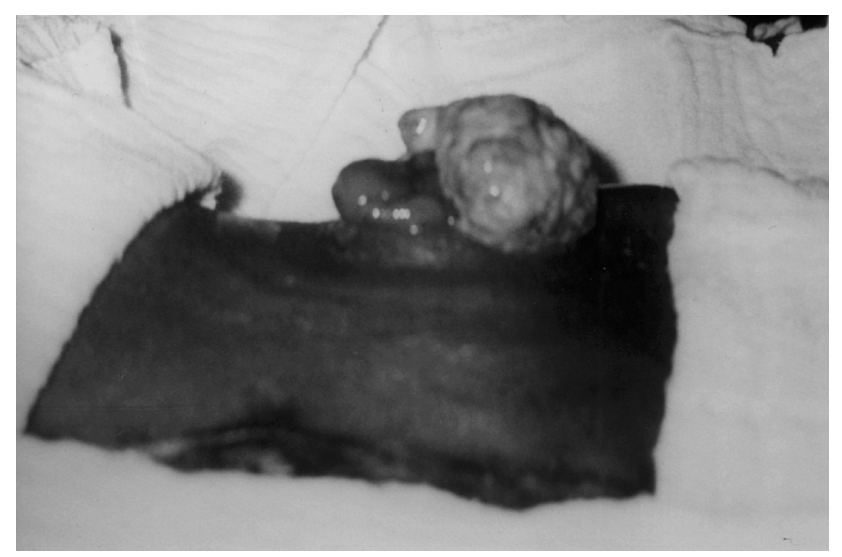

Figura 2
Frente a este achado, optou-se pela realização do tratamento cirúrgico local associado ao suporte clínico oncológico. Realizou-se a ressecção local da tumoração na colostomia, cujas dimensões eram de 4,7 x 3,5 x 3,0 centímetros, com o diagnóstico anatomopatológico de adenocarcinoma moderadamente diferenciado metastático. A doente faleceu por evolução da doença dois meses após o procedimento.

Caso 2: GVP, 53 anos, sexo masculino, branco, aposentado. Há três meses foi submetido a sigmoidectomia com anastomose primária colorretal, seguida de colostomia em alça de proteção, devido a diverticulite aguda de sigmóide. No acompanhamento ambulatorial notou o surgimento de tumoração na colostomia, associado a episódios frequientes de sangramento, durante o último mês. Na inspeção local havia uma lesão polipóide de aproximadamente três centímetros. Foi investigada a presença de outras lesões por meio da realização de uma colonoscopia, cujo resultado foi considerado normal, não evidenciando outras lesões polipóides. Foi indicada a ressecção local da lesão, cujo exame anatomo-patológico concluiu tratar-se de um pólipo inflamatório, hiperplásico.

Posteriormente o doente retornou ao ambulatório para a programação do fechamento da colostomia, que se realizou após um mês da polipectomia.

Caso 3: TAF, 63 anos, sexo masculino, submetido à Cirurgia de Miles por adenocarcinoma de reto em 1993, associada à quimioterapia e radioterapia adjuvante, não retornando para acompanhamento por dez anos. Em 2003, apresentou-se com massa na parede abdominal em região para-colostômica, hérnia paracolostômica e uma lesão úlcero-vegetante intra-luminal localizada a seis centímetros da margem cutânea, sendo biopsiada por colonoscopia. O exame anatomopatológico concluiu tratar-se de adenocarcinoma moderadamente diferenciado. A tomografia computadorizada abdominal mostrou uma massa heterogênea, medindo 7,6 x 5,8 centímetros, comprometendo a parede abdominal para-colostômica, sem o comprometimento de órgãos intra-cavitários (Figura-3).

Submetido à ressecção ampla da neoplasia, incluindo a colostomia e a parede abdominal adjacente, cólon descendente e 2/3 distais do cólon transverso; com uma nova colostomia terminal no quadrante superior direito. O doente apresentou boa evolução, tendo realizado quimioterapia adjuvante e está atualmente assintomático. 


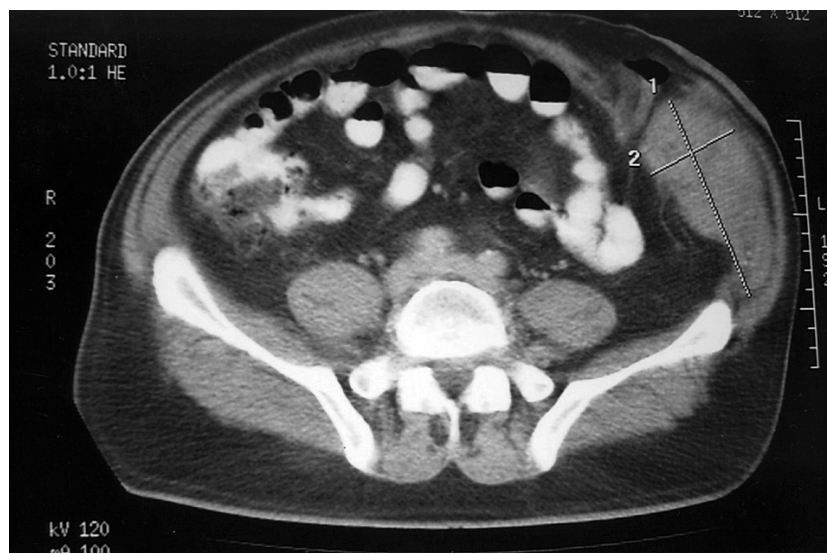

Figura 3

\section{DISCUSSÃO}

No cólon os carcinomas múltiplos podem ocorrer de maneira sincrônica ou metacrônica ${ }^{8}$, com uma incidência respectivamente, de 2 a $3 \%$ e de 3 a $5 \%{ }^{9}$. Considera-se como tumor metacrônico aquele que reincide no mesmo órgão ou em segmentos remanescentes que foram parcialmente ressecados, uma vez tendo encerrado o tempo considerado de cura do tumor inicial, que é de cinco anos. Entretanto, este período de tempo não é aceito completamente, podendo variar de dois a quatro anos o intervalo necessário para o aparecimento do segundo tumor, denominado metacrônico ${ }^{10}$.

Sabe-se que o tempo mínimo necessário para que ocorra a transformação de um pólipo adenomatoso em adenocarcinoma é de dois anos, portanto toda neoplasia desenvolvida neste período não deveria ser denominada como metacrônica, devendo ser definida como sincrônica ao primeiro tumor ${ }^{11}$.

A presença de carcinoma no sítio da colostomia não é freqüente; entretanto, o seu surgimento pode ocorrer ao longo do tempo e cursa com altos índices de morbi-mortalidade ${ }^{5,8}$.

Considerando que a colostomia é um segmento exteriorizado do cólon, com os mesmos fatores predisponentes e desencadeantes para o desenvolvimento de tumor colônico primário, admite-se que o risco neoplásico é semelhante à de qualquer outra porção do cólon, sendo significadamente elevado quando associado à lesão metacrônica ${ }^{12}$. Este fato ocorreu no caso 3, em que houve o aparecimento de nova lesão neoplásica junto à colostomia dez anos após a intervenção cirúrgica primária.

No caso 1, devido ao curto período de tempo existente entre a ressecção cirúrgica e o desenvolvimento da neoplasia no sitio da colostomia, devemos considerá-la como uma lesão sincrônica.

A recorrência de uma lesão pode ocorrer pelo desenvolvimento de um novo tumor (metacrônico), como consequiência de uma ressecção incompleta, pelo implante cirúrgico de células malignas na linha de anastomose ou em superfícies cruentas ${ }^{6}$, pela associação de lesão sincrônica ${ }^{5}$, e secundária ao trauma local imposto à mucosa colônica ${ }^{4,8}$. Pode haver um período de latência de 20 a 25 anos após a realização da colectomia para o surgimento da neoplasia no sitio da colostomia ${ }^{13} \mathrm{e}$ fatores distintos, como observado nos três casos, em que as causas da recorrência apresentavam etiologias diferentes.

O processo infamatório crônico encontrado na mucosa intestinal de doentes portadores de doença inflamatória, especialmente na retocolite ulcerativa inespecifica, é considerado a base estrutural para a degeneração maligna encontrada nos doentes portadores de ostomias ${ }^{4}$. Estes doentes são mais propensos às complicações colostômicas, assim como os portadores de polipose adenomatosa familiar, que apresentam na evolução adenoma-adenocarcinoma a possibilidade do desenvolvimento do carcinoma na ostomia ${ }^{14}$.

Atualmente o emprego difundido da colonoscopia, no estudo de doenças colorretais ou no acompanhamento pós-operatório de ressecções oncológicas, diagnosticando e removendo lesões pré-malignas, fez com que houvesse uma redução significativa na incidência do câncer colorretal metacrônico.

Clinicamente pode-se identificar a presença de tumoração local, sangramento intestinal e/ou obstrução intestinal no curso da recorrência tumoral no sitio da colostomia ${ }^{4}$, fato presente individualmente nos três casos descritos e que nos chamou a atenção para o correto diagnóstico clinico-cirúrgico.

Apresentamos três casos distintos de neoplasia da colostomia, com tratamentos particularizados em decorrência das características clínicas de cada doente e do exame anatomo-patológico. No primeiro caso, pelo fato da doente encontrar-se numa fase avançada da doença neoplásica (carcinomatose peritoneal), optamos pela ressecção local, objetivando apenas a melhora da função da colostomia, fato ocorrido no 
período pós-operatório de forma satisfatória. No segundo caso a realização de polipectomia foi adotada em virtude da característica benigna da lesão, que foi confirmada pelo exame anatomo-patológico, e também porque o doente encontrava-se no préoperatório de fechamento da colostomia, que ocorreu num breve período de tempo.

A conduta recomendada pela literatura, que é a da ressecção local ampla, incluindo a parede abdominal, com relocação da colostomia ${ }^{2,4,5,9,15}$, foi adotada no terceiro caso, com ampla ressecção dos folhetos músculo-aponeuróticos da parede abdominal, necessitando da colocação de prótese de polipropileno para a sua reconstrução, pois a lesão estava restrita ao sitio da colostomia.

A neoplasia de colostomia é um evento raro, cursando com pouca manifestação clínica, porém evidente e apresenta no seu diagnóstico precoce a melhor forma de controle da doença, cuja intervenção atenta do estomaterapeuta e do cirurgião, podem determinar uma baixa morbidade ao doente ostomizado, com melhor índice de sobrevida.

SUMMARY: Carcinomas rarely occur at the site of a colostomy. Colostomies are risk for malignancy, just as in any other portion of the colon. If the initial resection was for cancer, then the risk of metachronous colon adenocarcinoma is significantly higher than for the general population. Metachronous colorectal carcinoma occurs from 0.1 to 3.6 percent of patients who undergo surgery for colorectal carcinoma and the recurrence of the primary bowel malignancy that necessitated the stoma may present with signs of bleeding or obstruction. We report three cases of this rare neoplasia occurring at the site of a colostomy, and we review the literature.

Key words: Metachronous colorectal carcinoma, colostomy, stoma complications.

\section{REFERÊNCIAS BIBLIOGRÁFICAS}

1. Nour S, Beck J, Stringer MD. Colostomy complications: infants and children. Ann R Coll Surg Engl 1996;78: 526-30.

2. Shellito PC. Complications of abdominal stoma surgery. Dis Colon Rectum 1998;41:1562-72.

3. Park JJ, Del Pino A, Orsay CP, Nelson RL, Pearl RK, Cintron JR, Abcarian H. Stoma complications. Dis Colon Rectum 1999;42:1575-80.

4. Rothstein MS. Dermatologic considerations of stoma care. J Am Acad Dermatol 1986;15(3):411-32.

5. Corman ML; Intestinal stoma. In: Colon and Rectal Surgery. 4-ed., Los Angeles, 1998. pp.1264-1319.

6. Goligher JC, Duthie HL, Nixon HH. Tratamento do carcinoma do colo. In: Goligher JC, Duthie HL, Nixon HH, editores. Cirurgia do ânus, reto e colo. 5ed. São Paulo: Editora Manole; 1990. pp. 621-3.

7. Cruz GMG. Princípios técnicos da cirurgia dos estomas intestinais. In: Cruz GMG. Coloproctologia: terapêutica. Rio de Janeiro: Editora Revinter; 2000. p.579-607.

8. Shibuya T, Uchiyama K, Kokuma M, Shioya T, Watanabe Y, Moriyama Y, Matsumoto K, Yokosuka I. Metachronous adenocarcinoma occurring at a colostomy site after abdominoperineal resection for rectal carcinoma. J Gastroenterol2002;37:387-390.

9. Witz M, Lew S, Shpitz B, Griffel B, Dinbar A. Metachronous leiomyosarcoma in colostomy after abdominoperineal resection for rectal carcinoma. J Surg Oncol 1986; 32(4):200-2.
10. Cruz GMG. Câncer colorretal metacrônico: estudo da incidência cumulativa e apresentação de 10 casos pessoais. Rev bras ColoProct, 1996; 16(3):121-128.

11. Muto T, Bussey HJ, Morson BC. The evolution of cancer of the colon and rectum. Cancer 1975; 36:2251-70.

12. Carey PD, Suvarna SK, Baloch KG, Guilou PJ, Monson JR. Primary adenocarcinoma in an ileostomy: a late complication of surgery for ulcerative colitis. Surgery 1993; 113:712-5.

13. Gadacz TR, McFadden DW, Gabrielson EW, Ullah A, Berman JJ. Adenocarcinoma of the ileostomy: the latent risk of cancer after colectomy for ulcerative colitis and familial polyposis. Surgery 1990;107:698-703.

14. Saegusa K. A case of recurrent rectal carcinoma in the colostomy site. Chiba Med J 1986; 62:122.

15. Matos D, Cesaretti IUR. Complicações precoces e tardias dos estomas intestinais e urinários: aspectos preventivos e terapêuticos - estomas intestinais.In: Santos VLCG, Cesaretti IUR. Assistência em estomaterapia: cuidando do ostomizado. São Paulo: Ed. Atheneu; 2000. p.195-214.

Endereço para correspondência: VALDEMIR JOSÉ ALEGRE SALLES

Rua José Bonani, 199 - Bairro Independência 12.031-260 - Taubaté (SP)

E-mail: valiris@horizon.com.br 